\title{
Should tissue structure suppress or amplify selection to minimize cancer risk?
}

\author{
Laura Hindersin ${ }^{1}$, Benjamin Werner ${ }^{2}$, David Dingli ${ }^{3}$ and Arne Traulsen ${ }^{1 *}$
}

\begin{abstract}
Background: It has been frequently argued that tissues evolved to suppress the accumulation of growth enhancing cancer inducing mutations. A prominent example is the hierarchical structure of tissues with high cell turnover, where a small number of tissue specific stem cells produces a large number of specialized progeny during multiple differentiation steps. Another well known mechanism is the spatial organization of stem cell populations and it is thought that this organization suppresses fitness enhancing mutations. However, in small populations the suppression of advantageous mutations typically also implies an increased accumulation of deleterious mutations. Thus, it becomes an important question whether the suppression of potentially few advantageous mutations outweighs the combined effects of many deleterious mutations.
\end{abstract}

Results: We argue that the distribution of mutant fitness effects, e.g. the probability to hit a strong driver compared to many deleterious mutations, is crucial for the optimal organization of a cancer suppressing tissue architecture and should be taken into account in arguments for the evolution of such tissues.

Conclusion: We show that for systems that are composed of few cells reflecting the typical organization of a stem cell niche, amplification or suppression of selection can arise from subtle changes in the architecture. Moreover, we discuss special tissue structures that can suppress most types of non-neutral mutations simultaneously.

Reviewers: This article was reviewed by Benjamin Allen, Andreas Deutsch and Ignacio Rodriguez-Brenes. For the full reviews, please go to the Reviewers' comments section.

\section{Background}

It is a widely accepted view that tissues evolved to minimize the accumulation of somatic mutations during the live time of an individual [1-5]. Usually, this is achieved through the combined effect of multiple protective mechanisms. One important aspect is the hierarchical organization of most tissues, where few long lived stem cells give rise to a large and shorter lived population of progeny cells $[6,7]$. This allows for a high turnover of cells, while stem cells that are at risk to accumulate the potentially most harmful mutations, divide less frequently [1]. Thus most mutations occur at later stages of the hierarchy, where they are transient and are likely to be lost again due to the finite lifetime of most specialized cells [8]. The stem cell

\footnotetext{
*Correspondence: traulsen@evolbio.mpg.de

${ }^{1}$ Max Planck Institute for Evolutionary Biology, August-Thienemann-Str. 2, 24306 Plön, Germany

Full list of author information is available at the end of the article
}

pool may exhibit additional layers of protection, including a slow rate of replication, membrane pumps to rapidly secrete genotoxic agents, elevated DNA repair mechanisms, and feedback loops to maintain certain spatial organizations $[9,10]$.

Another important contribution to the suppression of mutations might arise from particular ways of spatial stem cell organization [1, 2]. Here, we discuss properties of such spatially organized systems and how the actual realization of the spatial organization needs to take extrinsic risk into account, for example the actual distribution of mutant fitness effects.

Our theoretical results are based on the Moran process on graphs [11]. A population of cells is located on a graph, where the links of a focal cell indicate the neighboring cells that can be replaced by the offspring of the focal cell. New mutations have a relative fitness $r>0$ compared to the wild-type with fitness 1 which influences their reproduction. One property of interest is the probability that a 
novel mutation takes over the whole population (reaches fixation on the graph). The graph represents the spatial structure of a population and is usually studied in comparison to a well-mixed population. Lieberman et al. defined a suppressor of selection as a graph that, compared to a well-mixed population, reduces the fixation probability of advantageous mutations (that have higher fitness than the wild-type) and increases the fixation probability of disadvantageous mutations (that have lower fitness than the wild-type) [11]. An amplifier is defined as the reverse: It increases the fixation probability of an advantageous mutation and decreases the fixation probability for a disadvantageous mutation, compared to the same mutation in a well-mixed population.

Often, it is implicitly assumed that suppressors of selection are desired, since they reduce the probability that a mutation that enhances the fitness of a cell reaches fixation within the stem cell population. This argument is at least partially the result of our limitation to reliably identify only strong drivers of selection in human malignancies. Although it has been known for a long time that some genomic alterations, such as mutations in the tumour suppressor genes TP53 and APC or the generation of fusion genes such as BCR-ABL are associated with specific tumors, often no known single driver mutation can be reliably identified. In these cases, either the driver oncogene is unknown, or the cancer phenotype is due to the combined effect of (many) mutations each with a small fitness effect. The current models of colorectal cancer development (adenoma to carcinoma sequence) or the progression of myelodysplastic syndromes to acute myeloid leukemia would be compatible with the latter model [12].

The properties of suppressors of selection have been the focus of research in several theoretical studies $[1,2,13,14]$. A suppressor of selection can reduce the probability that a strong driver mutation reaches fixation from values close to 1 to $1 / N$, where $N$ is the size of the population at risk, e.g. the number of stem cells in a colonic crypt. However, classical examples of suppressors of selection come with a trade off, as they increase the probability of fixation of disadvantageous mutations from almost 0 to $1 / N$. As many mutations in evolutionary biology lead to a reduced fitness, this poses the question whether stem cell organization should ideally suppress or amplify selection. If most mutations are advantageous and thus lead to a growth advantage, a suppressor of selection would reduce the rate of evolution. However, if most mutations are disadvantageous, an amplifier of selection ensures that these mutant cells cannot take over the population. This would prevent the successive accumulation of many deleterious mutations within stem cell populations and minimize the risk of tissue failure such as aplastic anemia.
Taking these conflicting considerations into account leads to our main question of which tissue architecture and population dynamics are optimal for minimizing cancer risk. The answer to this question depends on the stem cell population size, the precise stem cell organization and the distribution of fitness effects of both single mutants and mutants further ahead in the path towards the full cancer phenotype. In the following, we address these points. In Section Fixation of novel mutations we make an approximation for the rate of accumulation of mutations to compare the strongest suppressor of selection to the well-mixed population. Under the assumptions we make, the suppressor of selection reduces the accumulation of mutations if the total fraction of advantageous mutations is larger than $1 / N$. This first approximation leads to the question of how the distribution of fitness effects determines whether a suppressor or an amplifier of selection is useful to minimize cancer risk, which we study in Section The distribution of fitness effects of cancer mutations. In Section Population structures and their effect on fixation probabilities, we study small graphs exemplifying the stem cell population at the base of the colonic crypt. Since cancer is usually caused by the accumulation of several mutations, in Section Double mutations we ask which kind of structure would be optimal to prevent the fixation of two consecutive mutations.

\section{Fixation of novel mutations}

We first consider a well-mixed population of size $N$, where a cell's offspring can displace any other cell. In this case, the probability of fixation $\phi(r)$ of a single mutant cell that divides at rate $r>0$ when non-mutated cells divide at rate 1 is $[15,16]$

$$
\phi(r)=\frac{1-\frac{1}{r}}{1-\frac{1}{r^{N}}} .
$$

Throughout this work, we assume that the mutation rate is sufficiently small and the population size sufficiently large, such that we can consider one mutation at a time, i.e. we can neglect the effects of clonal interference [17-19]. Given a distribution of fitness effects $P(r)$ and a mutation rate $\mu$, the rate of accumulation of mutations in such a well-mixed population is

$$
\rho_{\mathrm{wm}}=\mu \int_{0}^{\infty} P(r) \phi(r) \mathrm{d} r .
$$

For simplicity, we first focus on large populations, $N \gg 1$, where we have

$$
\phi(r) \approx\left\{\begin{array}{l}
0 \text { for } r<1 \\
\frac{1}{N} \text { for } r=1 \\
1-\frac{1}{r} \text { for } r>1
\end{array}\right.
$$


Dividing the distribution of fitness effects into $P_{<}(r)$ for disadvantageous mutations and $P_{>}(r)$ for advantageous mutations, we obtain for the rate of accumulation of mutations

$$
\begin{aligned}
\rho_{\mathrm{wm}} & \approx \mu\left[\int_{0}^{1} P_{<}(r) 0 \mathrm{~d} r+\int_{1}^{\infty} P_{>}(r)\left(1-\frac{1}{r}\right) \mathrm{d} r\right] \\
& =\mu \int_{1}^{\infty} P_{>}(r) \mathrm{d} r-\mu \int_{1}^{\infty} \frac{P_{>}(r)}{r} \mathrm{~d} r .
\end{aligned}
$$

At most, the rate of accumulation of mutations is given by the mutation rate times the fraction of advantageous mutations, $\rho_{\mathrm{wm}} \leq \mu \int_{1}^{\infty} P_{>}(r) \mathrm{d} r$.

On the other hand, consider a hypothetical population structure which completely suppresses selection and leads to a neutral fixation probability which no longer depends on the selective advantage $r[2,11,16]$

$$
\phi(r)=\frac{1}{N} .
$$

For this structure, the rate of accumulation of mutations is

$$
\rho_{\mathrm{s}}=\frac{\mu}{N} \int_{0}^{\infty} P(r) \mathrm{d} r=\frac{\mu}{N} .
$$

Note that here, all mutations reach fixation with the same probability. The extreme suppressor of selection leads to a reduced accumulation of mutations compared to the well-mixed case if

$$
\int_{1}^{\infty} P_{>}(r) \mathrm{d} r>\frac{1}{N}
$$

i.e. if the total fraction of advantageous mutations is larger than $1 / N$.

Alternatively, we can think of a population that amplifies selection, i.e. that leads to a higher probability of fixation for beneficial (advantageous) mutations and to a lower probability of fixation for deleterious (disadvantageous) mutations. In the approximation used above, we neglected the fixation of deleterious mutations, because their fixation probability is zero for large $N$. For an amplifier of selection, the second term in Eq. 5 becomes smaller and the rate of accumulation of mutations increases, as advantageous mutations will reach fixation with an even higher probability. Therefore the fraction of beneficial mutations must be smaller than in a well-mixed population to decrease the accumulation rate $\rho$.

On the other hand, taking into account deleterious mutations, an amplifier of selection can ensure that these reach fixation with an even lower probability compared to a well-mixed population. For large populations this probability is already small and the additional effect will not be of much further interest. But in small populations, it may be crucial to ensure that these mutations are weeded out. Such small populations can be of relevance in cancer initiation. For example, the colon is subdivided into many colonic crypts, each of them being maintained by a small independent subset of stem cells [20-22].

With these first arguments, it becomes clear that the fraction of beneficial and deleterious mutations is important for answering the question whether an amplifier or a suppressor of selection is the optimal structure to minimize cancer risk.

\section{The distribution of fitness effects of cancer mutations}

In classical evolutionary biology, the distribution of fitness effects is of tremendous interest [23, 24]. The fitness effects of mutations depends on the evolutionary history of a population: If a population enters a novel environment, there can be mutations that lead to immediate fitness benefits. If a population has been evolving under constant conditions for a long time, it likely has already adapted to that specific environment and the chance that a novel mutation leads to beneficial effects decreases continuously. Usually, it is found that the vast majority of mutations are either deleterious or nearly neutral [25-27]. Only a small fraction of new mutations substantially increases evolutionary fitness.

Mutations that drive cancer initiation are usually thought to increase the fitness of a cell and only a few authors consider more general fitness landscapes [28]. Phenotypically these effects can be very diverse and include increased proliferation or decreased apoptosis rates, escape from an immune response or increased mutation rates $[9,22,29,30]$. In most theoretical studies, advantageous mutations are considered to have small constant effects on fitness. In contrast, Durrett et al. have addressed the case of randomly distributed fitness of mutations in a branching process [31]. However, the authors have focused on growing populations, which seems more appropriate for the evolution of an already seeded tumor rather than the tumor initiation process within a healthy tissue.

If only advantageous mutations are dangerous in the sense that they can lead to cancer, an ideal tissue should suppress the accumulation of such mutations. A universal mechanism of protection is to reduce the effective mutation rate, e.g. by developing effective DNA repair mechanisms and proofreading by DNA polymerases. This is highlighted by the increased risk of cancer in patients with inherited defects in DNA repair mechanisms. However, even the best DNA polymerases cannot completely eliminate the risk of mistakes and a few mutations will always occur. Another mechanism is to kill (hyper) mutated cells. This is the task of TP53, highlighted by the increased risk of early malignancy in people with an inherited defect in TP53 (e.g. Li Fraumeni syndrome). Unfortunately, overexpressing TP53, or introducing multiple copies of TP53 
in murine models leads to premature aging and actually, on average decreased life expectancy, although this may be a viable option in large, long-lived mammals such as elephants highlighting the enormous complexity and effects even of single genes [32]. There is the need for a balance between the mutation rate, DNA repair mechanisms and triggers of apoptosis within the cell to enable evolution of a species while reducing both the risk of cancer and early mortality. In addition, there might be alternative mechanisms that can suppress the spread of mutations within tissues. One such mechanism is the spatial organization of tissues.

Structures that suppress advantageous mutations usually also increase the fixation probability of mutations that cause reduced growth. In isolation, these mutations seem to be harmless because they are less fit than the wildtype cells. But they could interact with other subsequent mutations, leading to altered cell division properties via epistatic effects or environmental changes [28, 33, 34]. Such interactions between mutations have been investigated in experimental evolution in great detail [35-38], but they are usually neglected in the cancer community, partially because they are very difficult to measure. However, if initially disadvantageous mutations, which are arguably much more common, can turn to be dangerous for cancer initiation later, the organization of a tissue should adjust accordingly. An optimal tissue would in this scenario be an amplifier of selection, reducing the chances of fixation of the numerous disadvantageous mutations that can arise.

We study this by numerically calculating the fixation probability on an amplifier and suppressor graph of size 10. We use standard methods based on the transition matrix, which we generate from the adjacency matrix of the graph $[14,39,40]$. The transition matrix and the vector of fixation probabilities form a linear system of equations which can be solved for the fixation probabilities. To account for a broad range of fitness effects, we study mutations with fitness between 0 and 2, with 1 being the neutral reference fitness of the incumbent wild-type cells.

Figure 1 illustrates these results for two small graphs, a suppressor and an amplifier of selection. We highlight the areas where mutations with this fitness effect on the corresponding graph have a higher fixation probability than in a well-mixed population of the same size. Depending on the distribution of fitness effects, the rate of accumulation of new mutations can be minimized by either an amplifier or a suppressor of selection. If most novel mutations are deleterious, a suppressor of selection could lead to faster accumulation of mutations. On the other hand, if most mutations lead to an immediate growth advantage, a tissue that suppresses selection could be better for minimizing the accumulation of cancer mutations. According to these

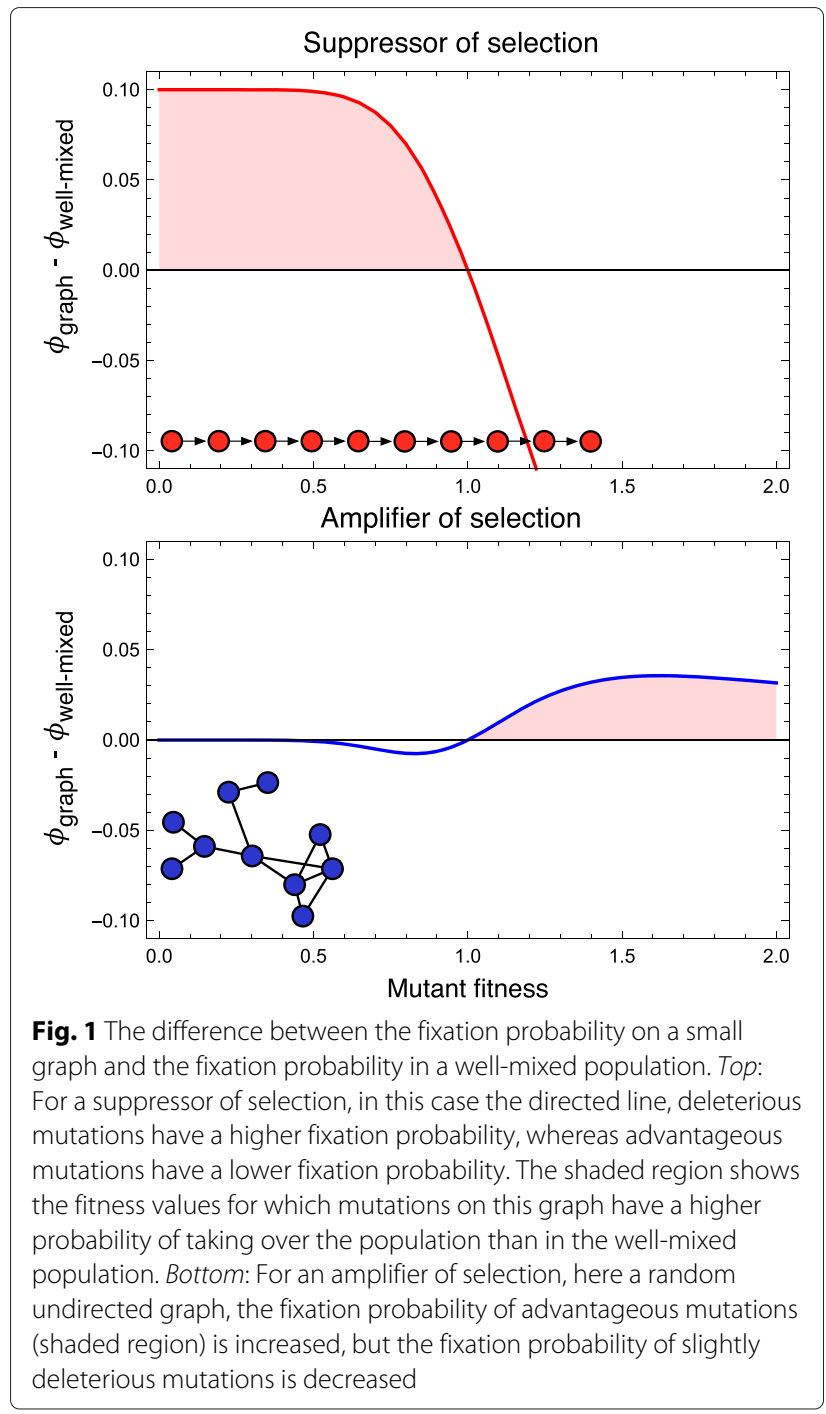

arguments, the distribution of fitness effects becomes a crucial quantity in answering the question whether a suppressor or an amplifier of selection leads to a minimal cancer risk.

\section{Population structures and their effect on fixation probabilities}

Ideally, a tissue would decrease the fixation probability of both beneficial and deleterious mutations. Previously we have shown that graphs which suppress both beneficial and deleterious mutations can be constructed for some update mechanisms [14]. One example is the cycle which suppresses both beneficial and deleterious mutations. There is evidence suggesting that the stem cells of the intestinal crypts replace each other in a onedimensional way similar to neighbors on a cycle $[4,41]$. However, the difference between the fixation probability 
on a cycle and a well-mixed population is relatively small and it remains unclear whether selection could lead to the evolution of such structures [42].

The simplest case of a structure that suppresses selection is a directed line [1]. The first cell is the root without incoming links and every cell can only replace its immediate successor. Mutations can only take over the whole graph if they occur in the root. Such a structure runs the risk of lineage extinction since there would be no redundancy in the system. One can envisage scenarios where this could lead to tissue failure and therefore place the organism at risk. It is perhaps for this reason that several stem cells occupy the base of each crypt in the colon.

To model the structure of colonic crypts, we consider small graphs that resemble this three-dimensional, bowllike structure [21, 22]. The lowest layer of nodes corresponds to the stem cells in the bottom of the crypt. Links between nodes determine which cells can replace each other. With directed and weighted links, one can account for the outflowing cell dynamics by which the colonic epithelium is replenished. The properties of such a population structure depend strongly on the details of its implementation.

We consider two updating mechanisms: (i) Birth-death, where a cell is chosen for reproduction based on its fitness and replaces one of its neighbors with an identical copy of itself and (ii) death-Birth, where a random cell dies and its neighbors compete based on their fitness to replace the empty site with their offspring cell. Both update mechanisms can have different biological motivation $[13,43,44]$. It is still an ongoing discussion whether birth-death or death-birth updating is a more accurate description of cell dynamics in colonic crypts. In biology they are sometimes referred to as pushing or pulling, where the signal of proliferation comes either from the stem cells directly or is induced by feedback mechanisms from differentiated cells.

Graphs that suppress selection for both update mechanisms are rare, as most random undirected graphs are suppressors of selection for death-Birth updating, but amplifiers of selection for Birth-death updating [14]. Figure 2 shows four small graphs, which could represent a

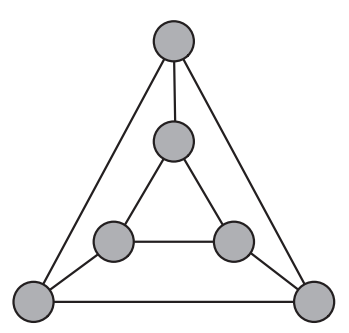

C

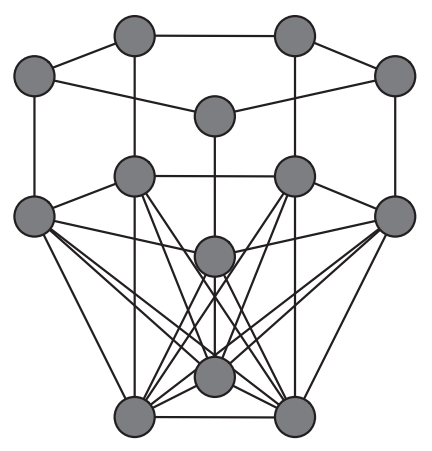

b

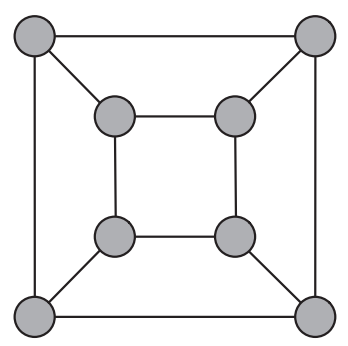

d

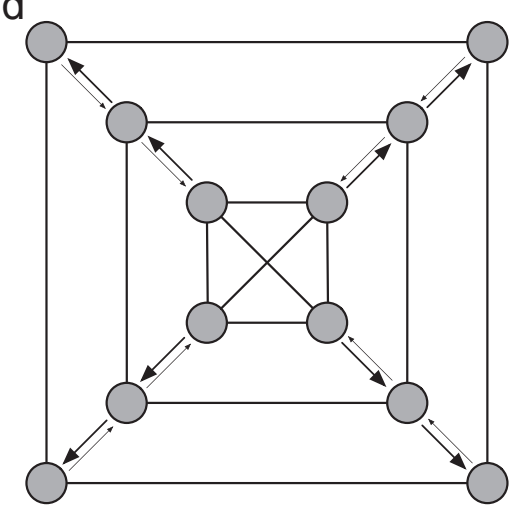

Fig. 2 Different graph structures that can model colonic crypts. a, b Two examples of suppressors of evolution for death-Birth updating. For Birth-death updating, these are equivalent to the well-mixed population in terms of the fixation probability. These two graphs consist of two layers of rings, their qualitative features do not change if we increase these structures to two rings of five, six, up to ten nodes. c This bowl-like graph with 13 nodes comprised of a base of 3 interconnected nodes, which are all connected to all nodes of the middle layer of five nodes. From the middle layer, every node has a corresponding node in the upper ring, to which it is connected. The links are undirected and unweighted. This graph is a suppressor of selection for both Birth-death and death-Birth updating. Thus, it reduces the fixation probabilities for advantageous mutations. $\mathbf{d}$ This graph has 12 nodes that are positioned in three layers. Here, the edges are directed and weighted. The outgoing edges between layers have a relative weight of 0.9 , whereas the corresponding incoming weights are 0.1 . This is to account for the outflowing cell-replacement of the colonic crypt. All other edge weights are 1. This graph is a suppressor of selection for Birth-death and death-Birth updating. The outflowing dynamics makes the suppression even stronger than in the same graph with unweighted edges 
the bottom part of a crypt, with different properties with respect to reducing or increasing fixation probabilities.

Figure $2 \mathrm{a}, \mathrm{b}$ shows two examples of graphs which are suppressors of evolution for death-Birth updating [14]. Since all nodes have the same number of neighbors, mutants on these graphs have the same fixation probability as the well-mixed population for Birth-death updating (Isothermal Theorem, proven in [11]). However, these graphs reduce the fixation probability of both advantageous and deleterious mutations for death-Birth updating. Here, we neglect the walls of the crypt and only model the bottom by two rings. However, it seems reasonable to ignore the crypt walls, as there is an outwards replacement of cells [20] and the fixation of a mutation within the crypt bottom implies fixation within the whole crypt.

The bowl-like graph illustrated in Fig. 2c suppresses selection for both Birth-death and death-Birth updating. Therefore, advantageous mutations have a lower fixation probability, but disadvantageous mutations have a higher fixation probability than the wild-type cells.

In Fig. 2d, we implement directed and weighted links to model the outward replacement dynamics of the crypt bottom. The graph becomes an even stronger suppressor of selection compared to the same graph with undirected and equally weighted links.

All fixation probabilities have been calculated numerically based on the method described in $[14,40]$. This approach is based on the numerical evaluation of the transition matrix of the Markov process associated with a graph, which naively scales with the graph size $N$ as $2^{N} \times$ $2^{N}$. This allows us to obtain numerically exact results, but restricts the analysis to relatively small graphs (currently, for our implementation [40] up to 23 nodes).

These examples illustrate that it is far from obvious how tissues should be structured to prevent the accumulation of mutations.

\section{Double mutations}

The initiation of cancer typically requires the accumulation of multiple mutations since a single mutated oncogene is rarely sufficient to cause cancer $[9,45,46]$. Thus, tissue architecture ultimately needs to prevent the accumulation of multiple mutations within single cells. Next, we study how a tissue should be structured to prevent the fixation of two consecutive mutations of different fitness effects. We assume that these mutations appear independently. Whether an amplifier or a suppressor of selection is more effective at preventing the accumulation of double mutations depends on the individual fitness effects of these mutations.

For example, consider the strongest possible suppressor of selection, e.g. the directed line. A mutated cell has a probability of $1 / N$ to take over, independent of its fitness relative to the wild type cells. For simplicity, we focus on a single mutational path, i.e. we consider only a single order of mutations. The probability for two independent consecutive mutations is thus $1 / N^{2}$. Let us compare this probability to that in a well-mixed population of the same population size. We study a system where the first mutation has relative fitness $r_{1}$ and competes against the wild-type cells of fitness 1 . The second mutation then has relative fitness $r_{2}$ and competes in a population of cells with fitness $r_{1}$. In this case, the combined fixation probability of the double mutant is

$$
\rho_{1 \rightarrow r_{1}} \rho_{r_{1} \rightarrow r_{2}}=\frac{1-\frac{1}{r_{1}}}{1-\left(\frac{1}{r_{1}}\right)^{N}} \frac{1-\frac{r_{1}}{r_{2}}}{1-\left(\frac{r_{1}}{r_{2}}\right)^{N}} .
$$

We now focus on mutations with small effects, $r_{1} \approx 1$ and $r_{2} \approx r_{1}$, and we expand around $r_{1}=r_{2}=1$,

$$
\begin{aligned}
\rho_{1 \rightarrow r_{1}} \rho_{r_{1} \rightarrow r_{2}} & \approx\left(\frac{1}{N}+\frac{\left(r_{1}-1\right)(N-1)}{2 N}\right)\left(\frac{1}{N}+\frac{\left(\frac{r_{2}}{r_{1}}-1\right)(N-1)}{2 N}\right) \\
& =\frac{1}{N^{2}}+\frac{N-1}{2 N^{2}}\left[r_{1}+\frac{r_{2}}{r_{1}}-2+\frac{N-1}{2}\left(r_{1}-1\right)\left(\frac{r_{2}}{r_{1}}-1\right)\right] .
\end{aligned}
$$

To see whether the directed line is more effective than the well-mixed population at preventing double mutations, we have to compare this to $1 / N^{2}$. The directed line leads to a lower overall fixation probability if

$$
\left(r_{1}+\frac{r_{2}}{r_{1}}-2\right)+\frac{N-1}{2}\left(r_{1}-1\right)\left(\frac{r_{2}}{r_{1}}-1\right)>0 .
$$

For two consecutive advantageous mutations, $1<r_{1}<$ $r_{2}$, this is clearly fulfilled and the directed line would reduce the fixation probability. In contrast, if the combined effect of two mutations is neutral $\left(r_{1} r_{2}=1\right)$, but the first mutation confers either an advantage or a disadvantage, the fixation probability for the directed line is larger than the fixation probability of the well-mixed population, as the second, negative term always outweighs the first term for $N>3$.

In general, the fixation probability of double mutants can either be larger or smaller on the directed line compared to the well-mixed populations. As illustrated in Fig. 3, this directly depends on the choice of fitness values. In the shaded areas of Fig. 3, the directed line is worse at preventing double mutations than the well-mixed population.

For many cases a strong suppressor of selection is worse at preventing the fixation of double mutations than the well-mixed population. If both mutations are advantageous, the directed line decreases the fixation probability. This effect prevails when one step is slightly disadvantageous, but as soon as the trajectory has to proceed through a sufficiently large fitness maximum or minimum, a well-mixed population performs better and suppresses 

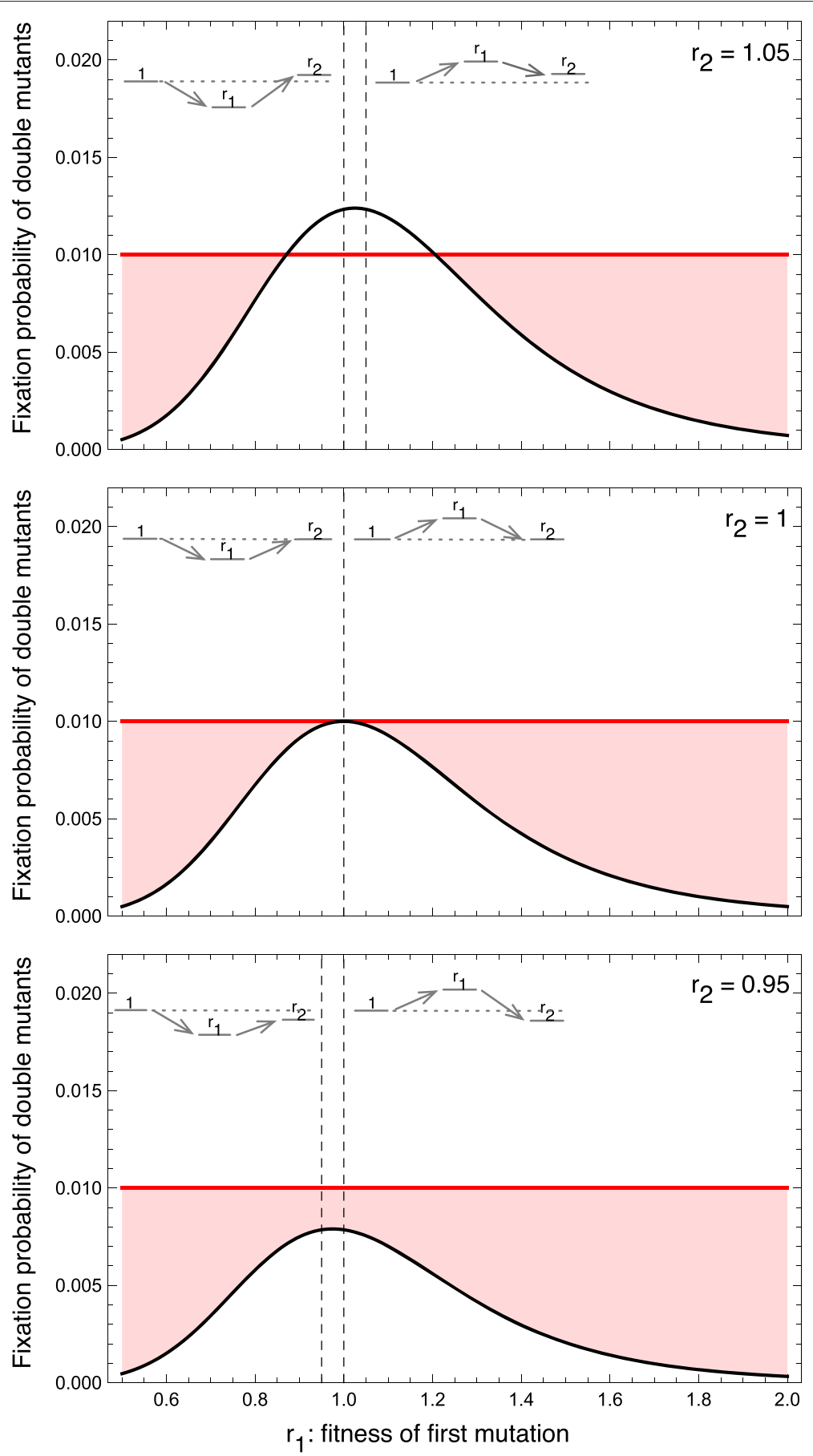

Fig. 3 Fixation probability of two consecutive mutations on the directed line, a suppressor of selection (red), and the well-mixed population (black) of size $N=10$. This fixation probability is plotted against the fitness of the first mutation. From top to bottom we show an advantageous second mutation $\left(r_{2}=1.05\right)$, a neutral $\left(r_{2}=1\right)$ and a disadvantageous $\left(r_{2}=0.95\right)$ second mutation. The shaded regions show the fitness values for which the fixation probability of the double mutations is higher on the directed line than in the well-mixed population

such double mutants more efficiently. These results show that the term "suppressor of selection" can be misleading, because in some cases the "suppressor" actually accelerates the fixation of double mutations.

\section{Discussion}

During homeostasis, stem cell replacement in the intestinal crypts is neutral [41]. Mutations that are commonly found in colorectal cancers likely give a competitive 
advantage to the cells [22]. This raises the question of how the tissue structure could act as a suppressor of novel mutations, decreasing the chance of advantageous mutations to take over the crypt.

Many graphs either act as a suppressor or amplifier of selection, preventing the fixation of either advantageous or disadvantageous mutations (compared to a well-mixed population), but not both [11, 13, 14, 47, 48].

However, these models have additional features that are not trivial: If mutations occur with a constant probability per division, then they do not arise with the same probability at all nodes. Instead, the arise with a probability proportional to the number of neighboring cells. In that case, a cycle-like structure with outflowing Birthdeath cell replacement suppresses both beneficial and deleterious mutations [42].

The question of optimal tissue organization in order to minimize cancer risk is very complex. In general, the lifetime cancer risk is positively correlated with the number of stem cell divisions [49-51]. However, every tissue has unique needs and risks. As we have shown, subtle changes in tissue architecture can profoundly change its protective properties. This might explain why different tissues have evolved towards different organizations, but also why these organizations can be compromised by different types of mutations.

Furthermore, it is far from obvious that oncogenic mutations necessarily confer a direct fitness advantage to a cell. Deleterious mutations might play a role in cancer initiation via epistatic effects [28,33]. Additionally, a mutated stem cell with lower replication rate could trigger its neighboring stem cells to compensate for the missing cell divisions by increased turnover and thus indirectly cause an increased cancer risk by effectively reducing the size of the active stem cell population.

Another risk is context dependent fitness, where the fitness of the mutant cell depends on the ecology (environment) of the cell population. For example, during development (growth) cell populations typically expand and favor mutant clones that grow faster, whereas in stationary conditions other phenotypes are selected for $[34,52,53]$. Thus, mutations that are neutral or disadvantageous at first, may become advantageous in later stages of development.

An additional effect of population structure is that the fixation of mutations becomes substantially slower in many graphs $[19,54]$, paving the way for increased clonal interference. It has been argued before that this will delay the accumulation of mutations and thus the onset of cancer [55].

We have shown that a "suppressor of selection" is not necessarily the optimal choice of a tissue structure in order to minimize cancer risk. It depends on the distribution of fitness effects. In order to prevent a sequence of two mutations, the directed line fares worse than the well-mixed population for most combinations of fitness values.

Overall, our approach is a step further to unravel the complexity of the spatial arrangement of a tissue to minimize the risk of cancer. A tissue architecture has to satisfy multiple requirements, some of which may appear to be conflicting. First and most importantly, it has to ensure the functionality of the organ. Secondly, it seems preferential to suppress harmful changes. Such changes can be manifold, and range from organ damage to cancer, normal or accelerated aging and possibly organ failure. We might have an intuitive understanding, why different organs evolved different architectures, and it seems natural that different architectures are prone to different errors. Nevertheless, our understanding of these problems is certainly incomplete and only recently has an evolutionary perspective become increasingly appreciated within the wider cancer research community. It is a hope that this might lead to a better understanding and ultimately a better approach towards cancer therapies.

\section{Reviewers' comments}

Reviewers' report 1: Benjamin Allen, Emmanuel College, Boston, USA

Summary This manuscript raises a set of interesting questions regarding the application of evolutionary graph theory to cancer initiation. It has been shown mathematically that some combinations of graph and update rule can amplify the effects of selection while others can suppress them. In applying this theory to cancer initiation in structured tissue, it is generally assumed that the goal is to suppress beneficial ("driver") mutations, because these can lead to runaway cell proliferation (cancer). This manuscript points out that it may also be important to reduce the fixation chances of deleterious mutations, because these mutations may also be important in tumor initiation. Overall, I think these are an important set of questions to raise. It does not convince me to abandon the hypothesis that tissues should evolve to suppress driver mutations, but it provides an interesting caveat to this hypothesis. The mathematics and the biology are both explained clearly. One of the most interesting points for me is the discussion after Eq. (11), that a directed line (which suppresses selection) could increase the fixation chances of a two-mutation sequence whose combined effect is neutral.

Recommendations Major comment: The manuscript rightly points out that either beneficial or deleterious mutations could be cancer initiators. The most important objective might therefore be to reduce the fixation chances of *all* mutations, including neutral ones. Allen 
et al. (2015) showed that this can happen under Bd updating if mutations occur with a constant probability per division. The accumulation of neutral mutations is slowed if the population is "fed" by a set of slow- dividing cells. I think this is also an important point to raise in discussions of how tissue architecture can affect tumor initiation (of course, I am biased since it is my paper).

Authors' response: Thank you for this positive evaluation of our work and for pointing out important aspects that remained unclear. We have rewritten and corrected all of these paragraphs. The major comment and the first minor issue are now discussed in the discussion and in the beginning of Section Population structures and their effect on fixation probabilities. We fully agree that our work does not imply that tissues should evolve to amplify selection, but our goal is to point out that a potentially important part of the process, the distribution of fitness effects, should be taken into account.

\section{Reviewers' report 2: Andreas Deutsch, TU Dresden, Germany}

Summary In this paper the authors investigate the interplay between the structure of tissues and the emergence of cancer-related mutations within these tissues. They focus on systems with a few cells to reflect the organization of stem cell niches and analyze whether the suppression or the amplification of selection contributes to minimize cancer risk. To address this question, first, the fixation of new mutations in a well-mixed population is investigated. Subsequently, the distribution of fitness effects of cancerrelated mutations is discussed and the importance of this distribution for cancer development is emphasized. The main argument for this conclusion is the comparison of the fixation probabilities of mutants on different structures in dependency of the mutant fitness. In the next section of the paper, four different graph structures that might be appropriate to model the structure of colonic crypts are introduced and different update mechanisms for the spread of mutants are investigated on these structures. Here, the authors want to illustrate that it is far from obvious how tissues should be structured to prevent the accumulation of mutations. Finally, to reflect the fact that cancer initiation usually requires multiple mutations, the fixation probability of two consecutive mutations in dependence of the underlying tissue structure and the fitness of the mutations is discussed.

Recommendations In my point of view, this manuscript exhibits some crucial weaknesses. A main critics is the overall structure of the manuscript. The introducing Background section provides only a very short and summarized overview of relevant previous works. Moreover, a clear question is not formulated so that it is hard for the reader to follow the subsequent sections. Furthermore, specific terms are used in different variations ("strong / weak mutations", "dangerous mutations") which are not introduced or explained. Another critics regarding the structure is the missing connection between the sections of the manuscript. Each section seems to address a new subquestion, but there is no attribution of these subquestions to a main question.

The manuscript also exhibits biological and mathematical inaccuracies. For example, approximations that are utilized are not discussed (Eqs. (4), (10)). The numerical methods that are used in Section Population structures and their effect on fixation probabilities should be shortly introduced and not only cited (lines 149-155). Some formulations are incorrect from a biological point of view ("mutations such as TP53, APC or BCR-ABL" / "In classical evolutionary biology, the distribution of fitness effects is a crucial quantity.").

The manuscript is very conceptual. However, the connection between biology and modeling remains largely unclear. The authors state that they concentrate on only a few cells to reflect the organization of stem cell niches. For which concrete tissues are the results of the manuscript applicable? In which case is it then sensible to assume a well mixed population like in Section Fixation of novel mutations? What is the biological mechanism behind the different used update mechanisms ("Birth-death, deathBirth") in Section Population structures and their effect on fixation probabilities? Unfortunately, these questions are not addressed or discussed.

Authors' response: We understand from these comments and those of the other reviewers that we have implicitly tailored the manuscript to a very specific audience and that our way of arguing may appear confusing. We are grateful that you pointed this out, as it would have a detrimental effect on the perception of this work. Part of the problem was the unclear structure. To broaden the Background section, we have introduced a paragraph about the Moran process on graphs. We have added an overview of the sections at the end of the Background connecting the questions addressed in the sections to the main question of the paper. Additionally, we have added summarizing sentences to the sections to bring the focus back to the main question and smoothen the transition between sections.

In Section The distribution of fitness effects of cancer mutations we have briefly introduced the numerical method used here and in Section Population structures and their effect on fixation probabilities. We prefer not to go into detail as we have referenced to a very detailed method paper [40]. By briefly introducing the method at this point before Fig. 1, we aim to draw a clear distinction between biological background and new results.

The concrete tissue to which our results are applicable is the colon consisting of many crypts where stem cells reside at the bottom. This is mentioned at several positions in 
the manuscript accompanied by citations of recent experimental papers that have shown these dynamics. In the beginning of Section Population structures and their effect on fixation probabilities we have added a paragraph about experimental evidence suggesting a cycle-like replacement behavior of these intestinal stem cells. A well-mixed population is always used as the reference case.

We have added more detail to the description of the update mechanisms ("Birth-death, death-Birth") in Section Population structures and their effect on fixation probabilities and the underlying biological mechanisms. As discussed in the references we point to, it is still an ongoing debate which mechanism is more accurately describing the biological process of cell replacement.

\section{Reviewers' report 3: Ignacio Rodriguez-Brenes, University of California, Irvine, USA}

Summary I found the article very interesting. It touches upon important questions that require more attention from the scientific community. For these reasons, I recommend the article for publication.

Recommendations Minor Recommendations: I have a few comments/questions that could enhance the readability of the manuscript. I believe most of them should be easily addressed.

I had trouble following the argument starting with "In the approximation used above..." [Line 41 and beyond]. This is an important point and I recommend rewriting it to make it clearer.

Figure 1 . The figure legend refers to the fixation probability; however, the label for the $y$-axis is rho, which before corresponded to the "the rate of accumulation of mutations" (Page 2, lines 45-46). Also, according to Eq. (2), rho should always be positive. I could be misinterpreting something, or there might a problem with the label of the axis, but either way it would be good to clarify these issues.

The article makes convincing arguments about the need to consider disadvantageous mutations (see e.eg. lines 8890, 97-99, and the Discussion Section). However, as it is written one potential reading of the discussion of TP53 (lines 78-85) is that the over-expression of this gene can lead to premature aging and a reduction of life-span in mice caused by the excessive (or overzealous) killing of mutated cells. TP53 has also a well-known role in sensing telomere length, which is different than its role on protecting against mutations and which could contribute to premature aging in mice that over-express this gene. Moreover, other mammalian species have many more copies of TP53 than mice (and humans) and are still able to sustain very long life spans (e.g. elephants). I therefore suggest a more nuanced discussion of the role of TP53.

In terms of references, the authors might find the article "Minimizing the risk of cancer: tissue architecture and cellular replication limits (2013)" interesting and related to the ideas discussed in their work.

Authors' response: Thank you for these very useful remarks. We have reworded the argument about the approximation. The y-axis in Fig. 1 has been corrected. Thank you for pointing this out. We also agree that our discussion of the role of TP 53 was insufficient in the previous version and have improved this.

\section{Acknowledgements}

We sincerely thank all three reviewers for their detailed and constructive feedback.

\section{Authors' contributions}

B.W., D.D., and A.T. have developed the concept. L.H. and A.T. have developed the analytical approach, L.H. has performed the numerical analysis. All authors have contributed to writing the manuscript. All authors have read and approved the manuscript.

\section{Competing interests}

The authors declare that they have no competing interests.

\section{Author details}

${ }^{1}$ Max Planck Institute for Evolutionary Biology, August-Thienemann-Str. 2, 24306 Plön, Germany. ${ }^{2}$ The Centre for Evolution and Cancer, The Institute of Cancer Research, 123 Old Brompton Road, London SW7 3RP, UK. ${ }^{3}$ Division of Hematology and Department of Molecular Medicine, Mayo Clinic, 200 First Street SW, Rochester, MN 55905, USA.

Received: 24 February 2016 Accepted: 4 June 2016

Published online: 23 August 2016

\section{References}

1. Nowak MA, Michor F, Iwasa Y. The linear process of somatic evolution. Proc Natl Acad Sci U S A. 2003;100:14966-9.

2. Michor F, Nowak MA, Frank SA, Iwasa Y. Stochastic elimination of cancer cells. Proc R Soc B. 2003;270:2017-24.

3. Komarova NL, Cheng P. Epithelial tissue architecture protects against cancer. Math Biosci. 2006;200:90-117.

4. Vermeulen L, Morrissey E, van der Heijden M, Nicholson AM, Sottoriva A, Buczacki S, Kemp R, Tavaré S, Winton D. Defining stem cell dynamics in models of intestinal tumor initiation. Science. 2013;342:995-8.

5. Bozic I, Nowak MA. Unwanted evolution. Science. 2013;342(6161):938-9.

6. Busch K, Klapproth K, Barile M, Flossdorf M, Holland-Letz T, Schlenner SM, Reth M, Höfer T, Rodewald HR. Fundamental properties of unperturbed haematopoiesis from stem cells in vivo. Nature. 2015;518(7540):542-6.

7. Rodriguez-Brenes IA, Wodarz D, Komarova NL. Minimizing the risk of cancer: tissue architecture and cellular replication limits. J R Soc Interface. 2013;10:20130410.

8. Werner B, Dingli D, Lenaerts T, Pacheco JM, Traulsen A. Dynamics of mutant cells in hierarchical organized tissues. PLoS Comput Biol. 2011;7: 1002290.

9. Hanahan D, Weinberg RA. The hallmarks of cancer. Cell. 2000;100:57-70.

10. Hanahan D, Weinberg RA. Hallmarks of cancer: the next generation. Cell. 2011;144(5):646-74.

11. Lieberman $E$, Hauert $C$, Nowak MA. Evolutionary dynamics on graphs. Nature. 2005;433:312-6.

12. Kandoth C, McLellan MD, Vandin F, Ye K, Niu B, Lu C, Xie M, Zhang Q, McMichael JF, Wyczalkowski MA, Leiserson MDM, Miller CA, Welch JS, Walter MJ, Wendl MC, Ley TJ, Wilson RK, Raphael BJ, Ding L. Mutational landscape and significance across 12 major cancer types. Nature. 2013;502:333-9. doi:10.1038/nature12634.

13. Kaveh K, Komarova NL, Kohandel M. The duality of spatial death-birth and birth-death processes and limitations of the isothermal theorem. J R Soc Open Sci. 2015;2:140465.

14. Hindersin L, Traulsen A. Most undirected random graphs are amplifiers of selection for birth-death dynamics, but suppressors of selection for death-birth dynamics. PLoS Comput Biol. 2015;11:1004437. 
15. Karlin S, Taylor HMA. A first course in stochastic processes, 2nd edition. London: Academic; 1975.

16. Nowak MA. Evolutionary dynamics. Cambridge MA: Harvard University Press; 2006.

17. Park SC, Krug J. Clonal interference in large populations. Proc Natl Acad Sci U S A. 2007;104(46):18135-40.

18. Campos PRA, Wahl LM. The effects of population bottlenecks on clonal interference, and the adaptation effective population size. Evolution. 2009;63(4):950-8.

19. Frean $M$, Rainey $P$, Traulsen $A$. The effect of population structure on the rate of evolution. Proc R Soc B. 2013;280:20130211.

20. Wright NA, Alison M. The Biology of Epithelial Cell Populations. New York: Oxford University Press, USA; 1984.

21. Baker AM, Cereser B, Melton S, Fletcher AG, Rodriguez-Justo M, Tadrous PJ, Humphries A, Elia G, McDonald SA, Wright NA, Simons BD, Jansen M, Graham TA. Quantification of crypt and stem cell evolution in the normal and neoplastic human colon. Cell Rep. 2014;8(4):940-7.

22. Vermeulen L, Snippert HJ. Stem cell dynamics in homeostasis and cancer of the intestine. Nat Rev Cancer. 2014;14:468-80.

23. Eyre-Walker A, Woolfit A, Phelps T. The distribution of fitness effects of new deleterious amino acid mutations in humans. Genetics. 2006;173: 891-900.

24. Eyre-Walker A, Keightley PD. The distribution of fitness effects of new mutations. Nature. 2007;8:610-8.

25. Zeyl C, DeVisser JA. Estimates of the rate and distribution of fitness effects of spontaneous mutation in Saccharomyces cerevisiae. Genetics. 2001;157:53-61.

26. Eyre-Walker A, Keightley PD, Smith NGC, Gaffney D. Quantifying the slightly deleterious mutation model of molecular evolution. Mol Biol Evol. 2002;19:2142-9.

27. Kassen R, Bataillon T. Distribution of fitness effects among beneficial mutations before selection in experimental populations of bacteria. Nat Genet. 2006;38:484-8.

28. Lipinski KA, Barber LJ, Davies MN, Ashenden M, Sottoriva A Gerlinger M. Cancer evolution and the limits of predictability in precision cancer medicine. Trends Cancer. 2016;2(1):49-63.

29. Beerenwinkel N, Antal T, Dingli D, Traulsen A, Kinzler KW, Velculescu VEE, Vogelstein B, Nowak MA. Genetic progression and the waiting time to cancer. PLoS Comput Biol. 2007;3:225.

30. Bozicl, Antal T, Ohtsuki H, Carter H, Kim D, Chen S, Karchin R, Kinzler KW, Vogelstein B, Nowak MA. Accumulation of driver and passenger mutations during tumor progression. Proc Natl Acad Sci U S A. 2010;107:18545-50.

31. Durrett R, Foo J, Leder K, Mayberry J, Michor F. Evolutionary dynamics of tumor progression with random fitness values. Theor Popul Biol. 2010;78(1):54-66. doi:10.1016/j.tpb.2010.05.001.

32. Abegglen LM, Caulin AF, Chan A, Lee K, Robinson R, Campbell MS, Kiso WK, Schmitt DL, Waddell PJ, Bhaskara S, Jensen ST, Maley CC, Schiffmann JD. Potential mechanisms for cancer resistance in elephants and comparative cellular response to dna damage in humans. JAMA. 2015;314(17):1850-60.

33. Bauer B, Siebert R, Traulsen A. Cancer initiation with epistatic interactions between driver and passenger mutations. J Theor Biol. 2014;358:52-60. doi:10.1016/j.jtbi.2014.05.018.

34. Werner B, Traulsen A, Dingli D. Ontogenic growth as the root of fundamental differences between childhood and adult cancer. Stem Cells. 2016;34:543-50.

35. Wolf JB, Brodie ED, Wade MJ. Epistasis and the evolutionary process. New York: Oxford University Press, USA; 2000.

36. Weinreich DM, Watson RA, Chao L. Perspective: sign epistasis and genetic constraint on evolutionary trajectories. Evolution. 2005;56(6):1165-74.

37. Khan Al, Dinh DM, Schneider D, Lenski RE, Cooper TF. Negative epistasis between beneficial mutations in an evolving bacterial population. Science. 2011;332(6034):1193-6. doi:10.1126/science.1203801.

38. de Visser JAGM, Cooper TF, Elena SF. The causes of epistasis. Proc Biol Sci. 2011;278(1725):3617-24.

39. Grinstead CM, Snell JL. Introduction to probability. Providence, RI: American Mathematical Society; 1997.

40. Hindersin L, Möller M, Traulsen A, Bauer B. Exact numerical calculation of fixation probability and time on graphs. arXiv. 2015;q-bio/1511.02696.
41. Lopez-Garcia C, Klein AM, Simons BD, Winton DJ. Intestinal stem cel replacement follows a pattern of neutral drift. Science. 2010;330(6005): 822-5.

42. Allen B, Sample C, Dementieva Y, Medeiros RC, Paoletti C, Nowak MA. The molecular clock of neutral evolution can be accelerated or slowed by asymmetric spatial structure. PLoS Comput Biol. 2015;11(2):1004108.

43. Zukewich J, Kurella V, Doebeli M, Hauert C. Consolidating birth-death and death-birth processes in structured populations. PLOS ONE. 2013;8(1): 54639.

44. Débarre F, Hauert C, Doebeli M. Social evolution in structured populations. Nat Commun. 2014;5(3409):4409.

45. Fearon ER, Vogelstein B. A genetic model for colorectal tumorigenesis. Cell. 1990;61:759-67.

46. Vogelstein B, Kinzler KW. Cancer genes and the pathways they control. Nat Med. 2004;10:789-99.

47. Adlam B, Nowak MA. Universality of fixation probabilities in randomly structured populations. Sci Rep. 2014;4:6692.

48. Adlam B, Chatterjee K, Nowak MA. Amplifiers of selection. Proc R Soc A. 2015;471(2181):20150114

49. Traulsen A, Pacheco JM, Luzzatto L, Dingli D. Somatic mutations and the hierarchy of hematopoiesis. BioEssays. 2010;32:1003-8.

50. Tomasetti C, Vogelstein B. Variation in cancer risk among tissues can be explained by the number of stem cell divisions. Science. 2015;347(6217): 78-81.

51. Noble R, Kaltz O, Hochberg ME. Peto's paradox and human cancers. Phil Trans R Soc B. 2015;370(1673):20150104.

52. Rozhok Al, Salstrom JL, DeGregori J. Stochastic modeling reveals an evolutionary mechanism underlying elevated rates of childhood leukemia. Proc Natl Acad Sci. 2016;113:1050-5. doi:10.1073/pnas.1509333113.

53. Werner B, Beier F, Hummel S, Balabanov S, Lassay L, Orlikowsky T, Dingli $D$, Brümmendorf TH, Traulsen A. Reconstructing the in vivo dynamics of hematopoietic stem cells from telomere length distributions. eLife. 2016;4:08687. doi:10.7554/eLife.08687.

54. Hindersin L, Traulsen A. Counterintuitive properties of the fixation time in network-structured populations. J R Soc Interface. 2014;11:20140606.

55. Martens EA, Kostadinov R, Maley CC, Hallatschek O. Spatial structure increases the waiting time for cancer. N J Phys. 2011;13. doi:10.1088/1367-2630/13/11/115014.

\section{Submit your next manuscript to BioMed Central and we will help you at every step:}

- We accept pre-submission inquiries

- Our selector tool helps you to find the most relevant journal

- We provide round the clock customer support

- Convenient online submission

- Thorough peer review

- Inclusion in PubMed and all major indexing services

- Maximum visibility for your research

Submit your manuscript at www.biomedcentral.com/submit
C Biomed Central 